\title{
Sonotubometry Findings in Patients with combined airway disease with and without Associated Eosinophilic Otitis Media
}

Ahmed Nasar, Mohammed Abd Allah, Mohammed Attia, Hasan Abd Al Naby

Department of ENT, Al Azhar University.

\begin{abstract}
Background: Active opening of the eustachian tube is accomplished by contraction of the paratubal muscles. Disturbance of any of the ET functions may contribute to the development of otitis media $(\mathrm{OM})$ with effusion and other middle ear diseases. Sonotubometry seems to be the most "physiologic" method for assessment of ET function and has the advantage that it can be performed on ears with an intact tympanic membrane and without the use of a pressure chamber.

The aim of this study was to compare the Eustachian tube function using sonotubometry in patients with combined airway disease associated with Eosinophilic otitis media with that having combined airway disease without otitis media.

Patients and methods: This study was applied on 45 patients divided into 3 groups each of 15.

$1^{\text {st }}$ group is the control group, $2^{\text {nd }}$ group patients with combined airway disease without Eosinophilic otitis media, $3^{\text {rd }}$ group patients with combined airway disease associated with Eosinophilic otitis media.

Results: Our results explains that the tubal opening durations were significantly longer in patients of EOM group than in patients with combined airway disease without EOM group and also the normal control group.

Conclusion: Sonotubometry can be performed in patients with or without an intact tympanic membrane and under physiological conditions. Sonotubometry is also inexpensive, painless, and easy to perform in both adults and children. Therefore, it has great potential value as a diagnostic tool for individuals with suspected ET disease.

Key words : Sonotubometry; eosionphilic Otitis media; Eustachian tube; airway disease
\end{abstract}

\section{Introduction}

The most important functions of the ET are protection, clearance, and ventilation of the middle ear (Bylander and stenstrom, 1998)\& (Sadé, 1997) Frequent active opening of the ET allows ventilation of the middle ear and equilibration of pressure changes. Active opening is accomplished by contraction of the paratubal muscles. The tensor veli palatini muscle plays the most important role. Contraction of the muscles occurs during swallowing, yawning, or movement of the mandible. Humans swallow approximately 1000 times a day, but not every swallowing action leads to ET opening with effective gas transfer into the middle ear (Bluestone et al., 1972)

Disturbance of any of the ET functions may contribute to the development of otitis media (OM) with effusion and other middle ear diseases (Straetemans et al., 2001), (Van Heerbeek et al., 2001).
Recently, there have been some reports of adult patients with intractable otitis media $(\mathrm{OM})$ with effusion (OME) or chronic OM (COM) associated with bronchial asthma. Those patients exhibit clinical characteristics markedly different from those of patients with common OM.

Those patients experience hearing loss for a prolonged period of time and sometimes become deaf gradually or suddenly.(Tomioka et al 1997), (Suzuki; et al 2004) Most of the patients also exhibited nasal polyposis or chronic rhinosinusitis, and some of them underwent nasal surgical procedures (Nagamine et al., 2002) (Suzuki et al., 2004)

In patients with bronchial asthma, a high prevalence of nasal polyposis is well documented (Settipane, 1996).

However; the incidence of EOM is lower than that of nasal polyposis in patients 
with asthma. The question is why some patients with asthma have EOM but others do not. The cause of OM is multifactorial, and one of the most important causes is eustachian tube dysfunction. In the present study, we evaluated the eustachian tube function of patients with asthma and with or without EOM and found that most of the patients with EOM had a patulous eustachian tube.

Sonotubometry seems to be the most "physiologic" method and has the advantage that it can be performed on ears with an intact tympanic membrane and without the use of a pressure chamber.

\section{Material and methods:}

Our study was applied on 45 patients divided into 3 groups each of 15

$1^{\text {st }}$ group is the control group which represents the normal individuals

$2^{\text {nd }}$ group patients with combined airway disease (having allergic rhino sinusitis with and without nasal polyposis with bronchial asthma) without

Eosinophilic otitis media (3cases were have bilateral allergic nasal polypi and functional endoscopic sinus surgery done for them 1month before doing the sonotubometry)

$3^{\text {rd }}$ group patients with combined airway disease (having allergic rhino sinusitis with and without nasal polyposis with bronchial asthma) associated with Eosinophilic otitis media

Sonotubometry done for patients of the $1^{\text {st }}$ and $2^{\text {nd }}$ group while sonotubometry with blood film for secretion taken from the middle ear to see the presence of Eosinophils and its numbers for the $3^{\text {rd }}$ group in order to confirm that the cause of middle ear effusion is allergic factors.

The patients age in all groups' were between 17 to 40 with mean age 26.27

Sonotubometry was done several times for the purpose of excluding false-negative data. And also effusion of the middle ear was also checked immediately before doing the test.

Sonotubometry measures the function of the ET using sound. A constant sound source is applied to the nostril while a microphone in the external auditory canal records the transmitted sound pressure level. The test is started with a constant sound signal in the nostril. The subject is asked to swallow (drink water); when the ET opens, an increase in sound level is measured. Thus, the active ventilatory function of the ET can be measured noninvasively. The measurements take place under physiological circumstances, without the use of extreme pressures and without the need for TM perforation. Thus, sonotubometry has several advantages and can be considered an appropriate method to measure ventilatory ET function.

Sonometry is the only method that studies the eustachian tube function in physiological conditions. Sonometry has been used by several authors. (Holmquist and Olen, 1980) (Murti et al., 1980) it records changes of sound intensities induced by the eustachian tube opening in the external meatus when a constant sound is applied in the nasal cavity. A major problem with this method was caused by environmental noises that polluted the signal recorded in the external auditory canal, (Magnuson and Falk, 1988) making possible only short-duration recordings of eustachian tube opening. This method was improved, however, by using high-frequency sounds (Virtanen, 1978) or by developing filters to decrease the signal-to-noise ratio. (Murti et al., 1980)

Sonometry studies the changes of sound intensities induced by the eustachian tube opening in the external meatus when a constant sound is applied in the nasal cavity. The function of the eustachian tube is not disturbed by the test procedure.

An original portable device with a high signal-to-noise ratio has been used. This device was able to detect a $6-\mathrm{dB}$ sound pressure level (SPL) signal in a $100-\mathrm{dB}$ SPL noise. Long duration recordings of auditory tubal openings could be performed. 


\section{Results:}

All measurements were easily performed, well tolerated, and successfully obtained.

Group (A): The tubal opening duration for these group were $(0.3,0.3,0.4,0.4,0.4,0.5,0.3$ $, 0.3,0.4,0.4,0.4,0.3,0.3,0.3,0.5$ ) as seen in table (2) and also Fig (3 and 4). The mean duration of tubal opening in this group is $0.3666 \mathrm{sec}$

\begin{tabular}{|c|c|c|c|c|c|c|c|c|c|c|c|c|c|}
\hline \multirow[t]{2}{*}{ 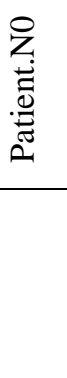 } & \multirow[t]{2}{*}{ 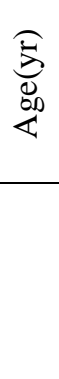 } & \multirow[t]{2}{*}{ 﨎 } & \multicolumn{4}{|c|}{ 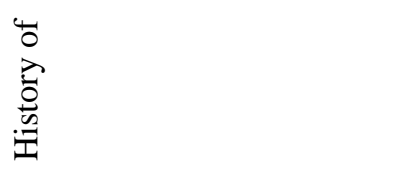 } & \multicolumn{6}{|c|}{ 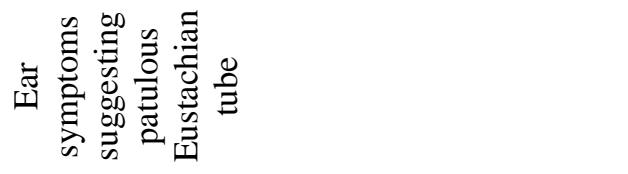 } & \multirow[t]{2}{*}{ 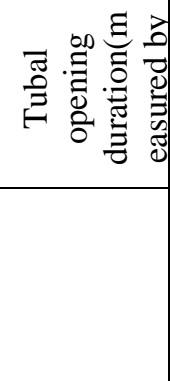 } \\
\hline & & & 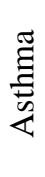 & 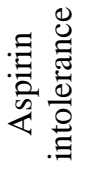 & $\begin{array}{l}\frac{9}{00} \\
\frac{\overrightarrow{0}}{2}\end{array}$ & 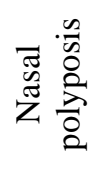 & 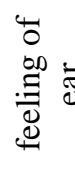 & 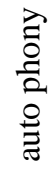 & 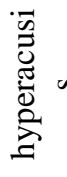 & 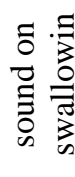 & 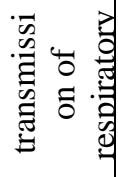 & 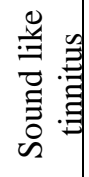 & \\
\hline $1-$ & 17 & $\mathrm{~F}$ & $-\mathrm{V}$ & $-\mathrm{V}$ & $-\mathrm{V}$ & $-\mathrm{V}$ & $-\mathrm{V}$ & $-\mathrm{V}$ & $-\mathrm{V}$ & $-\mathrm{V}$ & $-\mathrm{V}$ & $-\mathrm{V}$ & $0.2 \mathrm{sec}$ \\
\hline $2-$ & 19 & $\mathrm{~F}$ & $-\mathrm{V}$ & $-\mathrm{V}$ & $-\mathrm{V}$ & $-\mathrm{V}$ & $-\mathrm{V}$ & $-\mathrm{V}$ & $-\mathrm{V}$ & $-\mathrm{V}$ & $-\mathrm{V}$ & $-\mathrm{V}$ & $0.3 \mathrm{sec}$ \\
\hline $3-$ & 33 & $\mathrm{~F}$ & $-\mathrm{V}$ & $-\mathrm{V}$ & $-\mathrm{V}$ & $-\mathrm{V}$ & $-\mathrm{V}$ & $-\mathrm{V}$ & $-\mathrm{V}$ & $-\mathrm{V}$ & $-\mathrm{V}$ & $-\mathrm{V}$ & $0.1 \mathrm{sec}$ \\
\hline 4- & 24 & $\mathrm{~F}$ & $-\mathrm{V}$ & $-\mathrm{V}$ & $-\mathrm{V}$ & $-\mathrm{V}$ & $-\mathrm{V}$ & $-\mathrm{V}$ & $-\mathrm{V}$ & $-\mathrm{V}$ & $-\mathrm{V}$ & $-\mathrm{V}$ & $0.1 \mathrm{sec}$ \\
\hline $5-$ & 37 & M & $-\mathrm{V}$ & $-\mathrm{V}$ & $-\mathrm{V}$ & $-\mathrm{V}$ & $-\mathrm{V}$ & $-\mathrm{V}$ & $-\mathrm{V}$ & $-\mathrm{V}$ & $-\mathrm{V}$ & $-\mathrm{V}$ & $0.2 \mathrm{sec}$ \\
\hline 6- & 26 & $\mathrm{M}$ & $-\mathrm{V}$ & $-\mathrm{V}$ & $-\mathrm{V}$ & $-\mathrm{V}$ & $-\mathrm{V}$ & $-\mathrm{V}$ & $-V$ & $-\mathrm{V}$ & $-\mathrm{V}$ & $-\mathrm{V}$ & $0.2 \mathrm{sec}$ \\
\hline $7-$ & 29 & $\mathrm{~F}$ & $-\mathrm{V}$ & $-\mathrm{V}$ & $-\mathrm{V}$ & $-\mathrm{V}$ & $-\mathrm{V}$ & $-\mathrm{V}$ & $-\mathrm{V}$ & $-\mathrm{V}$ & $-\mathrm{V}$ & $-\mathrm{V}$ & $0.2 \mathrm{sec}$ \\
\hline $8-$ & 40 & $\mathrm{M}$ & $-\mathrm{V}$ & $-\mathrm{V}$ & $-\mathrm{V}$ & $-\mathrm{V}$ & $-\mathrm{V}$ & $-\mathrm{V}$ & $-\mathrm{V}$ & $-\mathrm{V}$ & $-\mathrm{V}$ & $-\mathrm{V}$ & $0.3 \mathrm{sec}$ \\
\hline 9- & 22 & $\mathrm{M}$ & $-\mathrm{V}$ & $-\mathrm{V}$ & $-\mathrm{V}$ & $-\mathrm{V}$ & $-\mathrm{V}$ & $-\mathrm{V}$ & $-\mathrm{V}$ & $-\mathrm{V}$ & $-\mathrm{V}$ & $-\mathrm{V}$ & $0.4 \mathrm{sec}$ \\
\hline $10-$ & 25 & $\mathrm{~F}$ & $-\mathrm{V}$ & $-\mathrm{V}$ & $-\mathrm{V}$ & $-\mathrm{V}$ & $-\mathrm{V}$ & $-\mathrm{V}$ & $-\mathrm{V}$ & $-\mathrm{V}$ & $-\mathrm{V}$ & $-\mathrm{V}$ & $0.4 \mathrm{sec}$ \\
\hline 11- & 30 & $\mathrm{M}$ & $-\mathrm{V}$ & $-\mathrm{V}$ & $-\mathrm{V}$ & $-\mathrm{V}$ & $-\mathrm{V}$ & $-\mathrm{V}$ & $-\mathrm{V}$ & $-\mathrm{V}$ & $-\mathrm{V}$ & $-\mathrm{V}$ & $0.2 \mathrm{sec}$ \\
\hline $12-$ & 19 & $\mathrm{M}$ & $-\mathrm{V}$ & $-\mathrm{V}$ & $-\mathrm{V}$ & $-\mathrm{V}$ & $-\mathrm{V}$ & $-\mathrm{V}$ & $-\mathrm{V}$ & $-\mathrm{V}$ & $-\mathrm{V}$ & $-\mathrm{V}$ & $0.3 \mathrm{sec}$ \\
\hline 13- & 18 & $\mathrm{M}$ & $-\mathrm{V}$ & $-\mathrm{V}$ & $-\mathrm{V}$ & $-\mathrm{V}$ & $-\mathrm{V}$ & $-\mathrm{V}$ & $-\mathrm{V}$ & $-\mathrm{V}$ & $-\mathrm{V}$ & $-\mathrm{V}$ & $0.3 \mathrm{sec}$ \\
\hline 14- & 23 & $\mathrm{~F}$ & $-\mathrm{V}$ & $-\mathrm{V}$ & $-\mathrm{V}$ & $-\mathrm{V}$ & $-\mathrm{V}$ & $-\mathrm{V}$ & $-\mathrm{V}$ & $-\mathrm{V}$ & $-\mathrm{V}$ & $-\mathrm{V}$ & $0.3 \mathrm{sec}$ \\
\hline $15-$ & 35 & $\mathrm{M}$ & $-\mathrm{V}$ & $-\mathrm{V}$ & $-\mathrm{V}$ & $-\mathrm{V}$ & $-\mathrm{V}$ & $-\mathrm{V}$ & $-V$ & $-\mathrm{V}$ & $-\mathrm{V}$ & $-\mathrm{V}$ & $0.2 \mathrm{sec}$ \\
\hline
\end{tabular}

Table: (1) represents. Summary of patients in the control group (A) 


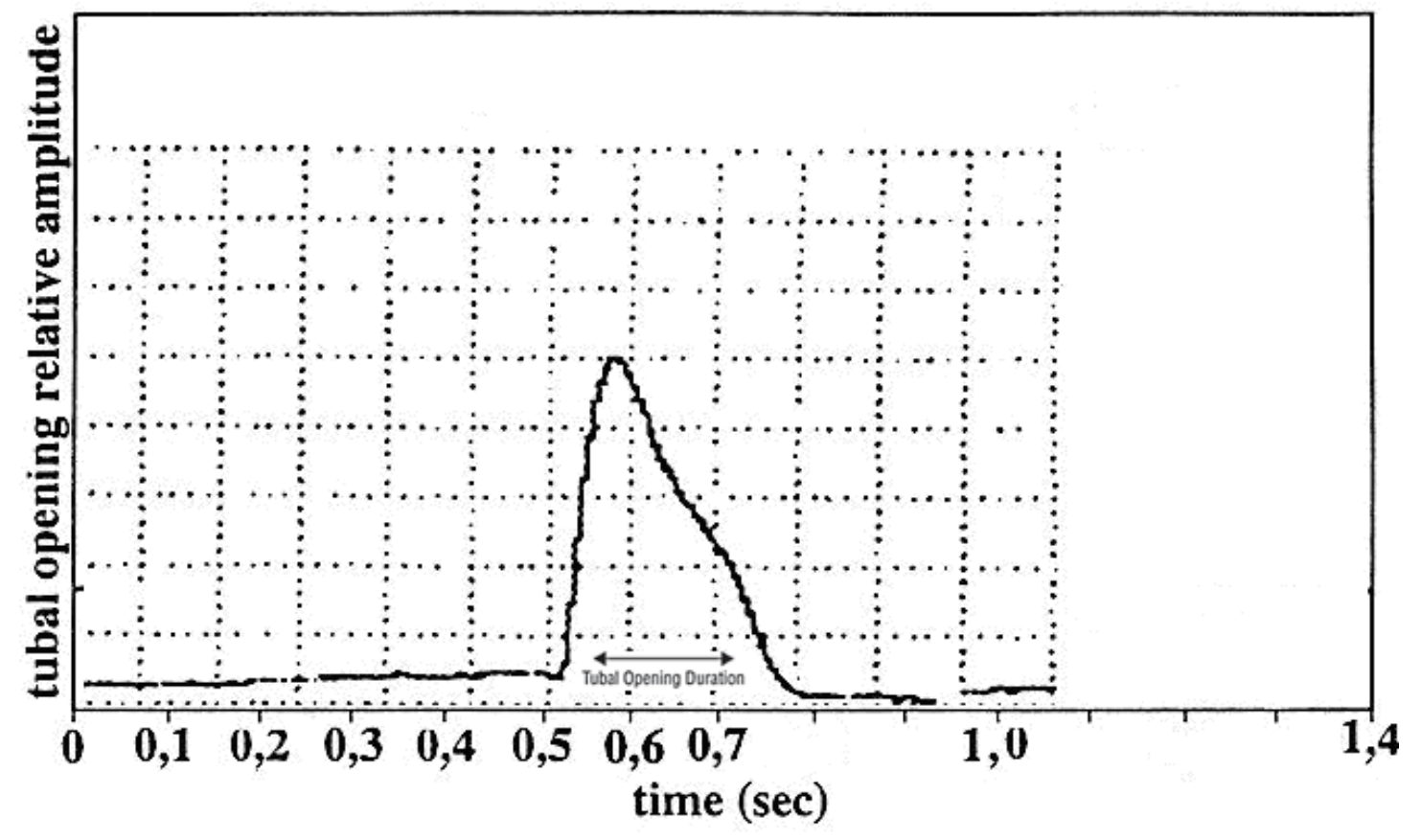

Fig (1): represents tubal opening duration for patient in group (A)

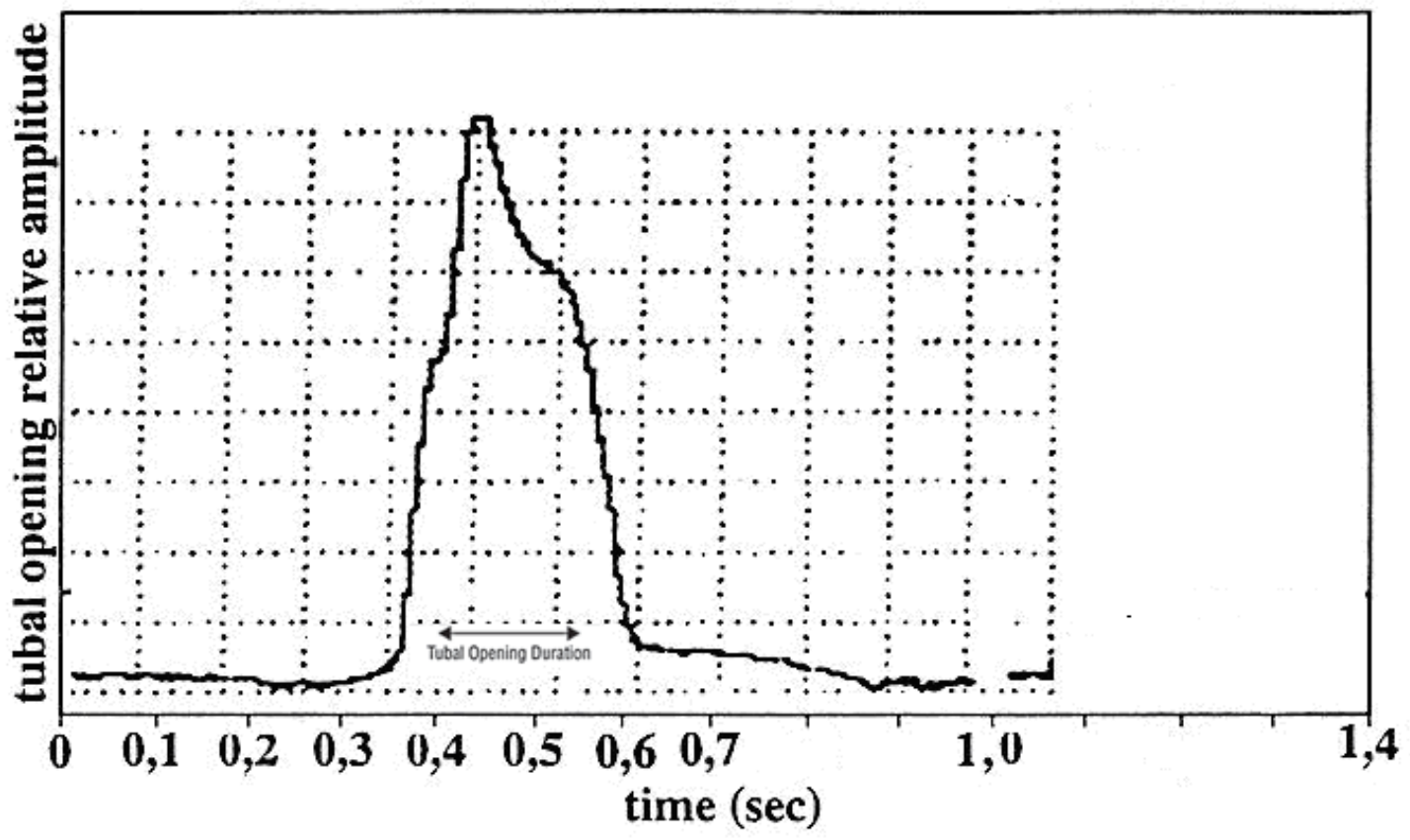

Fig (2): represents tubal opening duration for patient in group (A) 
Group (B): The tubal opening duration for these group were $0.2,0.3,0.1,0.1,0.2,0.2,0.2$ , $0.3,0.4,0.4,0.2,0.3,0.3,0.3,0.2$ ) as seen in table (1) and also Fig (1 and 2). The mean duration of tubal opening in this group is $0.2444 \mathrm{sec}$

\begin{tabular}{|c|c|c|c|c|c|c|c|c|c|c|c|c|c|}
\hline \multirow[t]{2}{*}{ 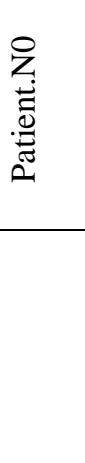 } & \multirow[t]{2}{*}{ 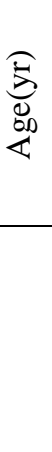 } & \multirow[t]{2}{*}{ 希 } & \multicolumn{4}{|l|}{ 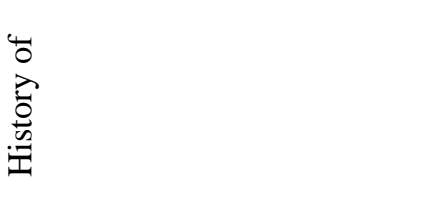 } & \multicolumn{6}{|c|}{ 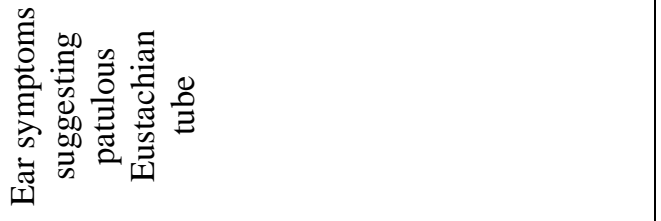 } & \multirow[t]{2}{*}{ 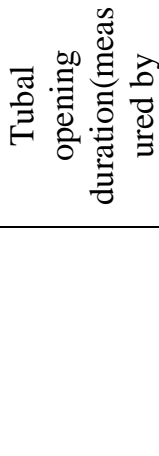 } \\
\hline & & & 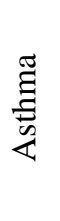 & 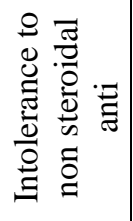 & 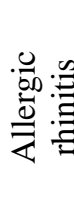 & 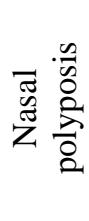 & 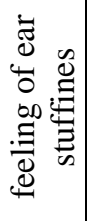 & 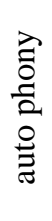 & 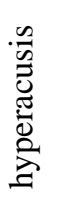 & 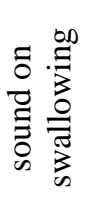 & 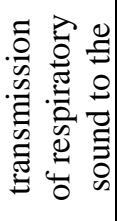 & 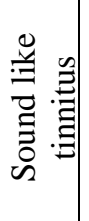 & \\
\hline $1-$ & 19 & $\mathrm{~F}$ & $+\mathrm{V}$ & $-\mathrm{V}$ & $+\mathrm{V}$ & $-\mathrm{V}$ & $-\mathrm{V}$ & $-\mathrm{V}$ & $-\mathrm{V}$ & $-\mathrm{V}$ & $-\mathrm{V}$ & $-\mathrm{V}$ & $0.3 \mathrm{sec}$ \\
\hline $2-$ & 21 & $\mathrm{~F}$ & $+\mathrm{V}$ & $-\mathrm{V}$ & $+\mathrm{V}$ & $-\mathrm{V}$ & $-\mathrm{V}$ & $-\mathrm{V}$ & $-\mathrm{V}$ & $-\mathrm{V}$ & $-\mathrm{V}$ & $-\mathrm{V}$ & $0.3 \mathrm{sec}$ \\
\hline $3-$ & 28 & $\mathrm{M}$ & $+\mathrm{V}$ & $-\mathrm{V}$ & $+\mathrm{V}$ & $-\mathrm{V}$ & $-\mathrm{V}$ & $-\mathrm{V}$ & $-\mathrm{V}$ & $-\mathrm{V}$ & $-\mathrm{V}$ & $-\mathrm{V}$ & $0.4 \mathrm{sec}$ \\
\hline 4- & 24 & $\mathrm{~F}$ & $+\mathrm{V}$ & $+\mathrm{V}$ & $+\mathrm{V}$ & $+\mathrm{V}$ & $-\mathrm{V}$ & $-\mathrm{V}$ & $-\mathrm{V}$ & $-\mathrm{V}$ & $-\mathrm{V}$ & $-\mathrm{V}$ & $0.4 \mathrm{sec}$ \\
\hline 5- & 39 & $\mathrm{~F}$ & $+\mathrm{V}$ & $+\mathrm{V}$ & $+\mathrm{V}$ & $+\mathrm{V}$ & $-\mathrm{V}$ & $-\mathrm{V}$ & $-\mathrm{V}$ & $-\mathrm{V}$ & $-\mathrm{V}$ & $-\mathrm{V}$ & $0.4 \mathrm{sec}$ \\
\hline 6- & 24 & $\mathrm{M}$ & $+\mathrm{V}$ & $-\mathrm{V}$ & $+\mathrm{V}$ & $-\mathrm{V}$ & $-\mathrm{V}$ & $-\mathrm{V}$ & $-\mathrm{V}$ & $-\mathrm{V}$ & $-\mathrm{V}$ & $-\mathrm{V}$ & $0.5 \mathrm{sec}$ \\
\hline 7- & 27 & $\mathrm{M}$ & $+\mathrm{V}$ & $-\mathrm{V}$ & $+\mathrm{V}$ & $-\mathrm{V}$ & $-\mathrm{V}$ & $-\mathrm{V}$ & $-\mathrm{V}$ & $-\mathrm{V}$ & $-\mathrm{V}$ & $-\mathrm{V}$ & $0.3 \mathrm{sec}$ \\
\hline 8- & 38 & $\mathrm{~F}$ & $+\mathrm{V}$ & $-\mathrm{V}$ & $+\mathrm{V}$ & $-\mathrm{V}$ & $-\mathrm{V}$ & $-\mathrm{V}$ & $-\mathrm{V}$ & $-\mathrm{V}$ & $-\mathrm{V}$ & $-\mathrm{V}$ & $0.3 \mathrm{sec}$ \\
\hline 9- & 23 & M & $+\mathrm{V}$ & $-\mathrm{V}$ & $+\mathrm{V}$ & $-\mathrm{V}$ & $-\mathrm{V}$ & $-\mathrm{V}$ & $-\mathrm{V}$ & $-\mathrm{V}$ & $-\mathrm{V}$ & $-\mathrm{V}$ & $0.4 \mathrm{sec}$ \\
\hline $10-$ & 25 & $\mathrm{~F}$ & $+\mathrm{V}$ & $-\mathrm{V}$ & $+\mathrm{V}$ & $-\mathrm{V}$ & $-\mathrm{V}$ & $-\mathrm{V}$ & $-\mathrm{V}$ & $-\mathrm{V}$ & $-\mathrm{V}$ & $-\mathrm{V}$ & $0.4 \mathrm{sec}$ \\
\hline $11-$ & 34 & M & $+\mathrm{V}$ & $-\mathrm{V}$ & $+\mathrm{V}$ & $+\mathrm{V}$ & $-\mathrm{V}$ & $-\mathrm{V}$ & $-\mathrm{V}$ & $-\mathrm{V}$ & $-\mathrm{V}$ & $-\mathrm{V}$ & $04 \mathrm{sec}$ \\
\hline $12-$ & 19 & M & $+\mathrm{V}$ & $-\mathrm{V}$ & $+\mathrm{V}$ & $-\mathrm{V}$ & $-\mathrm{V}$ & $-\mathrm{V}$ & $-\mathrm{V}$ & $-\mathrm{V}$ & $-\mathrm{V}$ & $-\mathrm{V}$ & $0.3 \mathrm{sec}$ \\
\hline 13- & 21 & $\mathrm{M}$ & $+\mathrm{V}$ & $-\mathrm{V}$ & $+\mathrm{V}$ & $-\mathrm{V}$ & $-\mathrm{V}$ & $-\mathrm{V}$ & $-\mathrm{V}$ & $-\mathrm{V}$ & $-\mathrm{V}$ & $-\mathrm{V}$ & $0.3 \mathrm{sec}$ \\
\hline 14- & 20 & $\mathrm{~F}$ & $+\mathrm{V}$ & $+\mathrm{V}$ & $+\mathrm{V}$ & $+\mathrm{V}$ & $-\mathrm{V}$ & $-\mathrm{V}$ & $-\mathrm{V}$ & $-\mathrm{V}$ & $-\mathrm{V}$ & $-\mathrm{V}$ & $0.3 \mathrm{sec}$ \\
\hline $15-$ & 40 & M & $+\mathrm{V}$ & $-\mathrm{V}$ & $+\mathrm{V}$ & $-V$ & $-\mathrm{V}$ & $-\mathrm{V}$ & $-\mathrm{V}$ & $-V$ & $-\mathrm{V}$ & $-\mathrm{V}$ & $0.5 \mathrm{sec}$ \\
\hline
\end{tabular}


Table: (2) represents. Summary of patients with combined airway disease without EOM group (B)

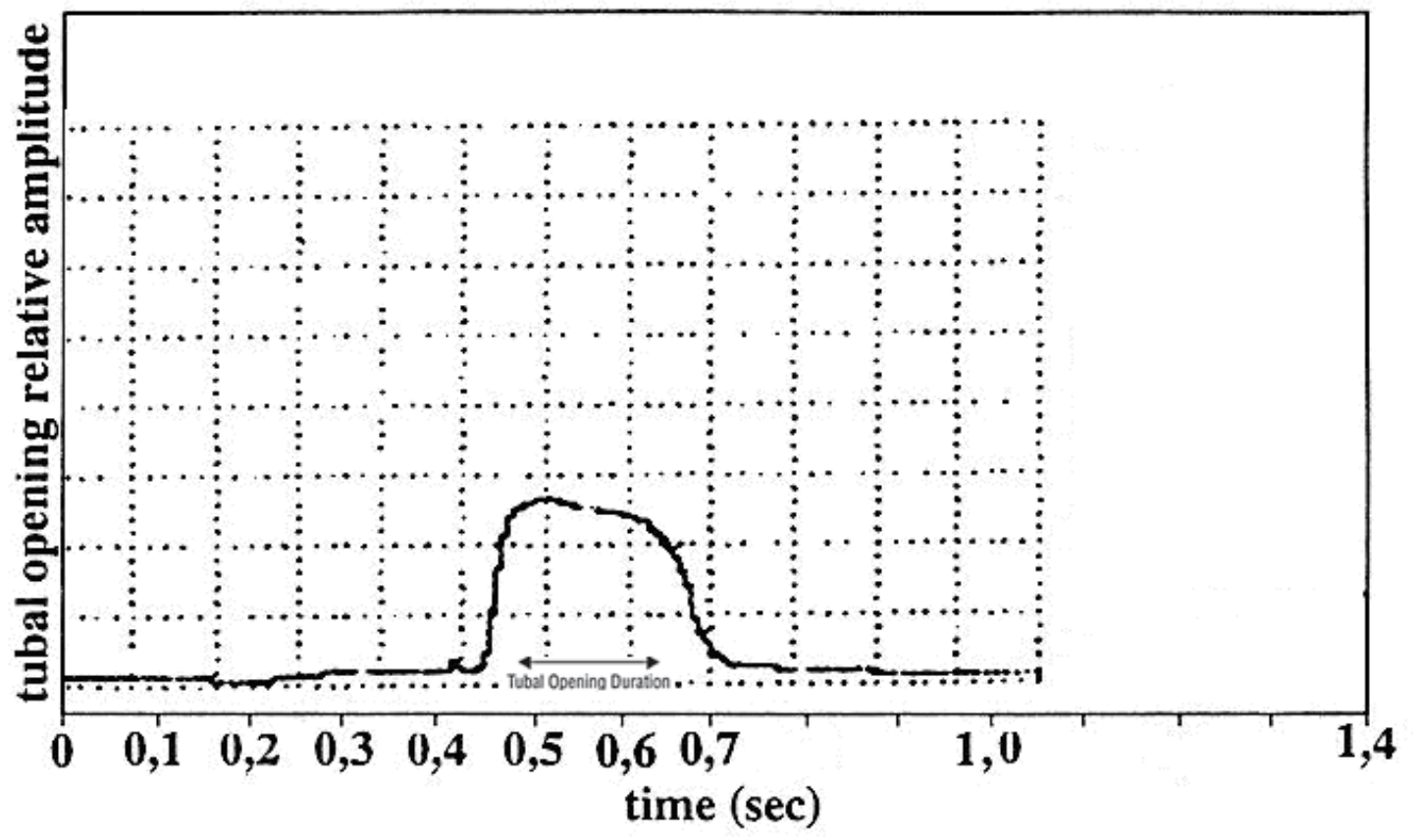

Fig (3): represents tubal opening duration for patient in group (B)

1.

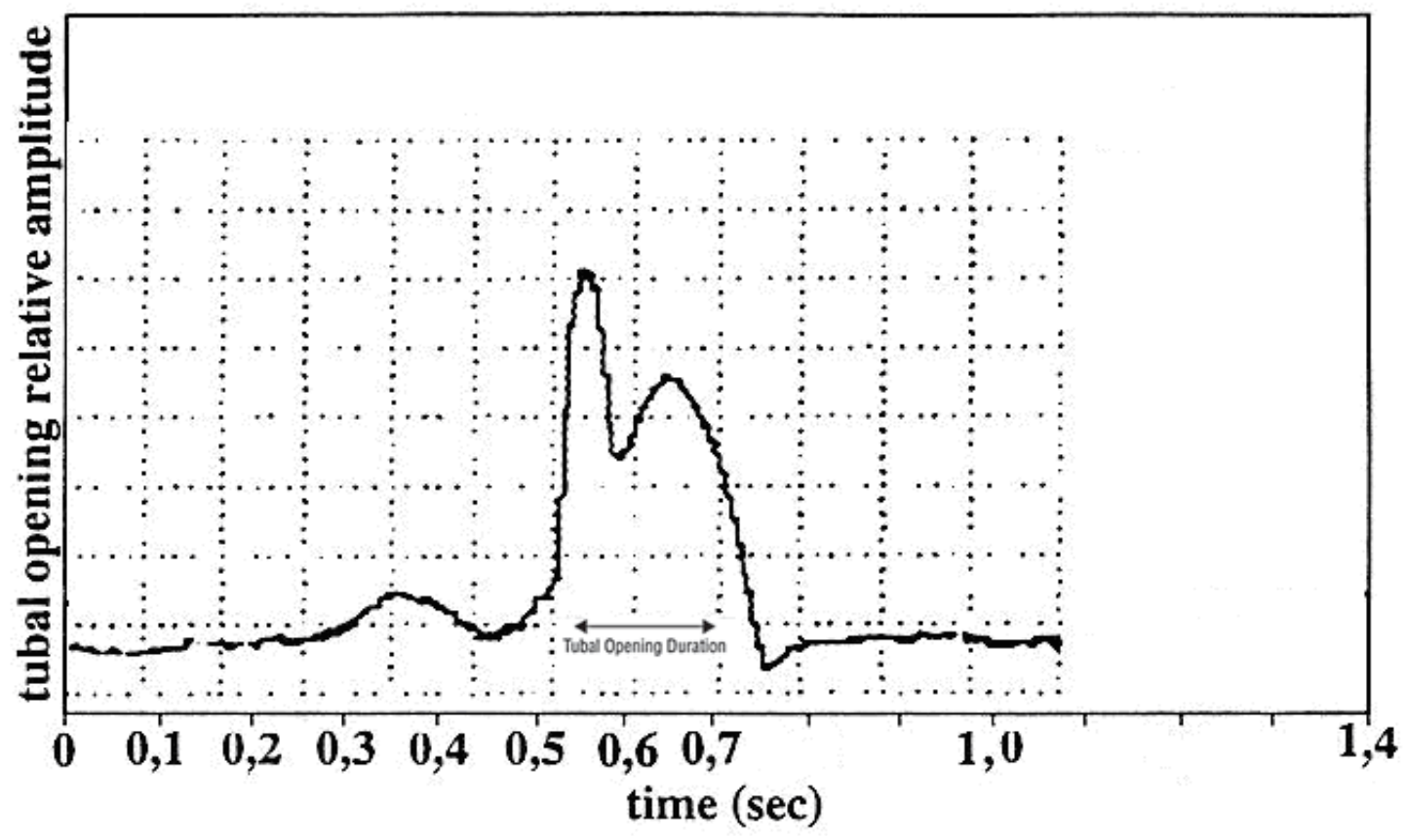

Fig (4): represents tubal opening duration for patient in group (B) 
Group (C): The tubal opening duration for these group were $(0.8,0.7,0.9,1.0,0.9,0.8,0.9$ , $0.7,1.2,1.1,1.4,0.9,1.1,1.0,1.1 \mathrm{sec}$ ) as seen in table (3) and also Fig (5). The mean duration of tubal opening in this group is $0.9666 \mathrm{sec}$

\begin{tabular}{|c|c|c|c|c|c|c|c|c|c|c|c|c|c|}
\hline \multirow[t]{2}{*}{$\begin{array}{l}\text { Patie } \\
\text { nt.NO }\end{array}$} & \multirow[t]{2}{*}{$\begin{array}{l}\text { Age } \\
(\mathrm{yr})\end{array}$} & \multirow{2}{*}{$\begin{array}{l}\mathrm{s} \\
\mathrm{e} \\
\mathrm{x}\end{array}$} & \multicolumn{4}{|c|}{ History of } & \multicolumn{6}{|c|}{$\begin{array}{l}\text { Ear symptoms suggesting patulous } \\
\text { Eustachian tube }\end{array}$} & \multirow{2}{*}{$\begin{array}{c}\text { Tubal } \\
\text { opening } \\
\text { duration( } \\
\text { measured } \\
\text { by } \\
\text { sonotubo } \\
\text { metry } \\
\end{array}$} \\
\hline & & & $\begin{array}{c}\text { Ast } \\
\text { hm } \\
\text { a }\end{array}$ & $\begin{array}{c}\text { Intoler } \\
\text { ance to } \\
\text { non } \\
\text { steroid } \\
\text { al anti } \\
\text { inflam } \\
\text { matory } \\
\text { drugs }\end{array}$ & $\begin{array}{l}\text { Alle } \\
\text { rgic } \\
\text { rhin } \\
\text { itis }\end{array}$ & $\begin{array}{c}\text { Nasa } \\
1 \\
\text { poly } \\
\text { posi } \\
\text { s }\end{array}$ & $\begin{array}{c}\text { feeli } \\
\text { ng } \\
\text { of } \\
\text { ear } \\
\text { stuff } \\
\text { ines }\end{array}$ & $\begin{array}{c}\text { aut } \\
\mathrm{o} \\
\mathrm{ph} \\
\text { on } \\
\mathrm{y}\end{array}$ & $\begin{array}{l}\text { hyper } \\
\text { acusis }\end{array}$ & $\begin{array}{c}\text { sound } \\
\text { on } \\
\text { swall } \\
\text { owin } \\
\text { g }\end{array}$ & $\begin{array}{l}\text { trans } \\
\text { missio } \\
\mathrm{n} \text { of } \\
\text { respir } \\
\text { atory } \\
\text { sound } \\
\text { to the } \\
\text { ear }\end{array}$ & $\begin{array}{c}\text { Sou } \\
\text { nd } \\
\text { like } \\
\text { tinn } \\
\text { itus }\end{array}$ & \\
\hline $1-$ & 20 & $\mathrm{~F}$ & $+\mathrm{V}$ & $-\mathrm{V}$ & $+\mathrm{V}$ & $+\mathrm{V}$ & $+\mathrm{V}$ & $+\mathrm{V}$ & $-\mathrm{V}$ & $-\mathrm{V}$ & $-\mathrm{V}$ & $+\mathrm{V}$ & $0.8 \mathrm{sec}$ \\
\hline $2-$ & 22 & $\mathrm{~F}$ & $+\mathrm{V}$ & $-\mathrm{V}$ & $+\mathrm{V}$ & $+\mathrm{V}$ & $-\mathrm{V}$ & $+\mathrm{V}$ & $+\mathrm{V}$ & $+\mathrm{V}$ & $-\mathrm{V}$ & $-\mathrm{V}$ & $0.7 \mathrm{sec}$ \\
\hline $3-$ & 27 & $\mathrm{M}$ & $+\mathrm{V}$ & $-\mathrm{V}$ & $+\mathrm{V}$ & $+\mathrm{V}$ & $-\mathrm{V}$ & $-\mathrm{V}$ & $+\mathrm{V}$ & $+\mathrm{V}$ & $+\mathrm{V}$ & $-\mathrm{V}$ & $0.9 \mathrm{sec}$ \\
\hline 4- & 30 & M & $+\mathrm{V}$ & $+\mathrm{V}$ & $+\mathrm{V}$ & $+\mathrm{V}$ & $-\mathrm{V}$ & $-\mathrm{V}$ & $+\mathrm{V}$ & $+\mathrm{V}$ & $-\mathrm{V}$ & $+\mathrm{V}$ & $1.0 \mathrm{sec}$ \\
\hline $5-$ & 19 & $\mathrm{~F}$ & $+\mathrm{V}$ & $+\mathrm{V}$ & $+\mathrm{V}$ & $+\mathrm{V}$ & $+\mathrm{V}$ & $+\mathrm{V}$ & $-\mathrm{V}$ & $-\mathrm{V}$ & $-\mathrm{V}$ & $+\mathrm{V}$ & $0.9 \mathrm{sec}$ \\
\hline 6- & 24 & $\mathrm{M}$ & $+\mathrm{V}$ & $-\mathrm{V}$ & $+\mathrm{V}$ & $-\mathrm{V}$ & $-\mathrm{V}$ & $-\mathrm{V}$ & $+\mathrm{V}$ & $+\mathrm{V}$ & $+\mathrm{V}$ & $-\mathrm{V}$ & $0.8 \mathrm{sec}$ \\
\hline $7-$ & 27 & $\mathrm{~F}$ & $+\mathrm{V}$ & $-\mathrm{V}$ & $+\mathrm{V}$ & $-\mathrm{V}$ & $-\mathrm{V}$ & $-\mathrm{V}$ & $-\mathrm{V}$ & $-\mathrm{V}$ & $-\mathrm{V}$ & $-\mathrm{V}$ & $0.9 \mathrm{sec}$ \\
\hline $8-$ & 30 & $\mathrm{~F}$ & $+\mathrm{V}$ & $-\mathrm{V}$ & $+\mathrm{V}$ & $-\mathrm{V}$ & $-\mathrm{V}$ & $-\mathrm{V}$ & $+\mathrm{V}$ & $+\mathrm{V}$ & $+\mathrm{V}$ & $-\mathrm{V}$ & $0.7 \mathrm{sec}$ \\
\hline 9- & 28 & $\mathrm{M}$ & $+\mathrm{V}$ & $-\mathrm{V}$ & $+\mathrm{V}$ & $-\mathrm{V}$ & $-\mathrm{V}$ & $-\mathrm{V}$ & $+\mathrm{V}$ & $+\mathrm{V}$ & $+\mathrm{V}$ & $+\mathrm{V}$ & $1.2 \mathrm{sec}$ \\
\hline $10-$ & 35 & $\mathrm{~F}$ & $+\mathrm{V}$ & $-\mathrm{V}$ & $+\mathrm{V}$ & $-\mathrm{V}$ & $-\mathrm{V}$ & $-\mathrm{V}$ & $+\mathrm{V}$ & $+\mathrm{V}$ & $+\mathrm{V}$ & $-\mathrm{V}$ & $1.1 \mathrm{sec}$ \\
\hline $11-$ & 3 & $\mathrm{M}$ & $+\mathrm{V}$ & $-\mathrm{V}$ & $+\mathrm{V}$ & $+\mathrm{V}$ & $-\mathrm{V}$ & $-\mathrm{V}$ & $+\mathrm{V}$ & $+\mathrm{V}$ & $+\mathrm{V}$ & $-\mathrm{V}$ & $1.4 \mathrm{sec}$ \\
\hline 12- & 17 & $\mathrm{M}$ & $+\mathrm{V}$ & $-\mathrm{V}$ & $+\mathrm{V}$ & $-\mathrm{V}$ & $+\mathrm{V}$ & $+\mathrm{V}$ & $-\mathrm{V}$ & $+\mathrm{V}$ & $-\mathrm{V}$ & $-\mathrm{V}$ & $0.9 \mathrm{sec}$ \\
\hline 13- & 26 & $\mathrm{M}$ & $+\mathrm{V}$ & $-\mathrm{V}$ & $+\mathrm{V}$ & $-\mathrm{V}$ & $-\mathrm{V}$ & $-\mathrm{V}$ & $+\mathrm{V}$ & $+\mathrm{V}$ & $-\mathrm{V}$ & $+\mathrm{V}$ & $1.1 \mathrm{sec}$ \\
\hline 14- & 22 & $\mathrm{~F}$ & $+\mathrm{V}$ & $+\mathrm{V}$ & $+\mathrm{V}$ & $+\mathrm{V}$ & $+\mathrm{V}$ & $+\mathrm{V}$ & $-\mathrm{V}$ & $-\mathrm{V}$ & $-\mathrm{V}$ & $-\mathrm{V}$ & $1.0 \mathrm{sec}$ \\
\hline 15- & 18 & $\mathrm{M}$ & $+\mathrm{V}$ & $-\mathrm{V}$ & $+\mathrm{V}$ & $-\mathrm{V}$ & $+\mathrm{V}$ & $+\mathrm{V}$ & $-\mathrm{V}$ & $-\mathrm{V}$ & $-\mathrm{V}$ & $+\mathrm{V}$ & $1.1 \mathrm{sec}$ \\
\hline
\end{tabular}

Table: (3) represents. Summary of patients of combined airway disease with EOM group $(\mathrm{C})$ 


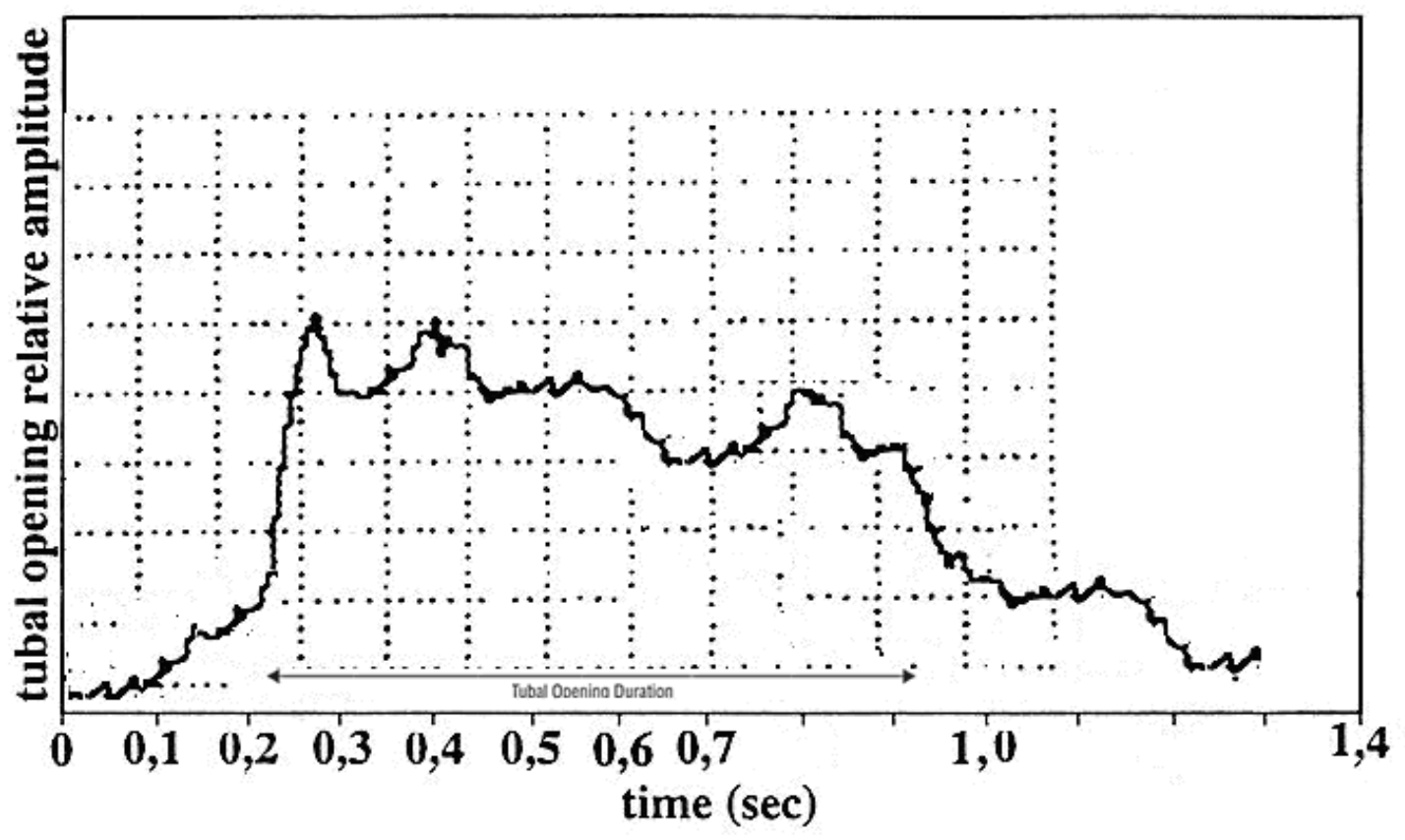

Fig (5): represents tubal opening duration for patient in group (C)

Our results explains that the tubal opening durations were significantly longer in patients of EOM group than in patients with combined airway disease without EOM group and also the normal control group(Fig 6). In addition, the opening area formed by sound pressure level(SPL) change were significantly larger in the EOM group than the other groups and no to much difference in the opening area between the non EOM group and the normal control group. Also in this study most patients of the EOM group did not detect an improvement in their symptoms after surgical or medical treatment of secretory otitis media and even after doing Valsalva maneuver indicating the presence of patulous Eustachian tube in patients with EOM . 


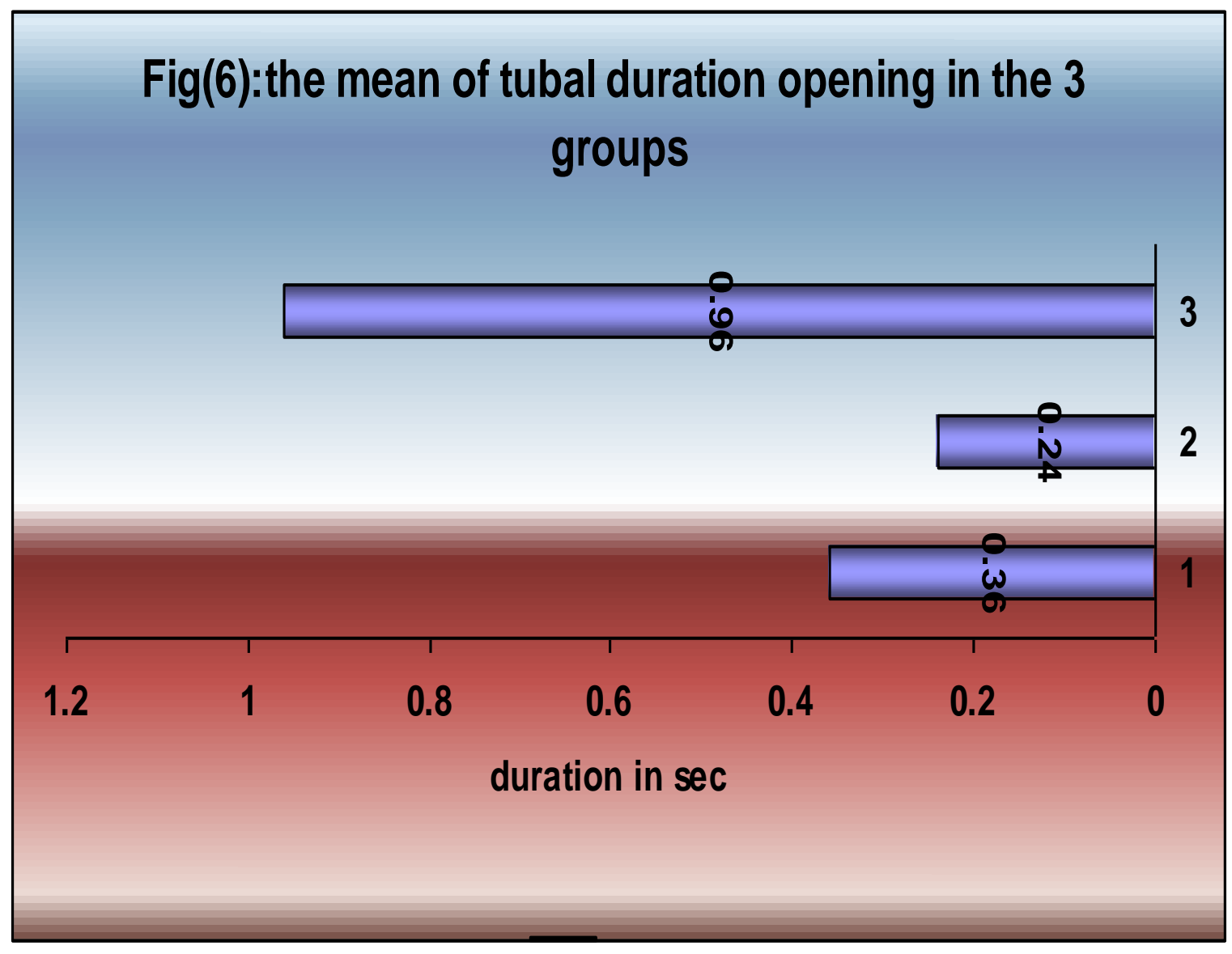

\section{Discussion:}

Several test methods are available to assess the ventilatory function of the ET. However, most of these tests have the disadvantage that they can only be performed on ears with a perforated tympanic membrane or a ventilation tube in situ. An even more important disadvantage is that these tests do not measure ventilatory ET function under physiological circumstances. Relatively large pressure changes (i.e., nonphysiological pressure changes) must be applied to evaluate the active ventilatory function. Furthermore, the inflationdeflation test can cause an uncomfortable feeling for the test subject.

In contrast, sonotubometry can be performed in patients with or without an intact tympanic membrane and under physiological conditions. Sonotubometry is also inexpensive, painless, and easy to perform in both adults and children. Therefore, it has great potential value as a diagnostic tool for individuals with suspected ET disease.

In this study, we determined the eustachian tube function of patients with asthma and with or without EOM by using sonotubometry and found that a patulous eustachian tube may predispose a patient to the disease which does not respond will to medical or surgical management

(Tsuji et al., 2003) reported that a history of $\mathrm{OM}$ is a causative factor for a patulous eustachian tube. They suggested the following hypothesis: after inflammation of the middle ear has ceased, fibrosis of the eustachian tube mucosa occurs, leading to a diseased, patulous eustachian tube. By contrast, (Virtanen and Palva 1982) reported that in 100 ears undergoing surgery for COM, about one third of the ears showed no tubal passage for sound during swallowing, $1 \%$ to $2 \%$ of the ears showed patulous patterns, and the remaining ears showed positive and normal patterns on findings from a 
sonotubogram. They concluded that

patulous eustachian tube is a rare condition in patients with COM. We also studied the eustachian tube function of the patients in the COM control group who had no active inflammation and found that most of them showed normal or slightly stenotic sonotubometric patterns, consistent with the results of (Virtanen and Palva 1982) In the present study, otitis media itself resulted in patulous Eustachian tube in patients of the EOM group so further studies is required to explains the cause of patulous Eustachian tube in patients with $\mathrm{OM}$ itself resulted in patulous eustachian tubes in the EOM group. Further studies should be performed to explain the cause of patulous eustachian tubes in patients with Eosinophilic otitis media.

It has been reported that in sonotubometry findings of healthy adults, $89.8 \%$ showed positive opening and closing patterns on voluntary swallowing and that the mean duration of tubal opening was (286.4) milliseconds (Okubo et al.,1984) In our study, for the normal control group the mean duration of tubal opening was 366.6 milliseconds.(Kakizaki et al., 2006) reported that significant difference in tubal opening duration for the asthmatic patients with EOM than patients with asthma but without EOM and this agree with our results also in the present study.

There are several diseases characterized by eosinophilic accumulation in the mucosa, such as bronchial asthma, eosinophilic pneumonia, and eosinophilic gastritis. In the upper respiratory tract, allergic rhinitis, allergic fungal sinusitis, (Ponikau et al., 1999) eosinophil dominant nasal polyposis, (Katzenstein et al., 1983) and eosinophilic mucin rhinosinusitis (Ferguson 2000) have recently been recognized as some of these conditions.

(Iino et al., 2001) These patients have highly viscous MEEs containing numerous eosinophils.

The middle ear symptoms are extremely resistant to conventional treatment for OME or COM, and the patients experience hearing loss for a prolonged period of time.

\section{Conclusion:}

Sonotubometry easily performed, well tolerated, successfully obtained, has great advantages over other function tests and may therefore be a very useful tool in monitoring and/or clinical research of ET dysfunction or function.

\section{References:}

1- Bluestone CD, Paradise JL. and Beery QC. (1972): Physiology of the eustachian tube in the pathogenesis and management of the middle ear effusions. Laryngoscope; 82:1654-70.

2- . Bylander Groth A. and Stenstrom C. (1998): Eustachian tube function and otitis media in children. Ear Nose Throat J; 77:7629.

3- Ferguson BJ. (2000): Eosinophilic mucin rhinosinusitis: a distinct clinicopathological entity. Laryngoscope.; 110:799-813.

4- Holmquist J. and Olen L. (1980): Evaluation of the ET function. J Laryngol Otol;94:15-23.

5- Kakizaki K. Saruya S. Katano H. Komiya T. Kodera K. and Ohta K. (2006): Archives of Otolaryngology -- Head \& Neck Surgery. 132(10):1109-14.

6- Katzenstein AL, Sale SR. and Greenberger PA. (1983): Allergic Aspergillus sinusitis: a newly recognized form of sinusitis. J Allergy Clin Immunol.; 72:89-93.

7- Iino Y, Nagamine H, Yabe T. and Matsutani S. (2001): Eosinophils are activated in middle ear mucosa and middle ear effusion of patients with intractable otitis media associated with bronchial asthma. Clin Exp Allergy. 31:1135-1143.

8- Magnuson B. and Falk B.(1988): Physiology of the eustachian tube and middle ear pressure regulation. In: Sade J, ed. Physiology of the Ear. New York: Raven Press;81-101.

9- Murti KG, Stern RM, Cantekin EI. and Bluestone CD. (1980):Sonometric evaluation of eustachian tube function using broadband stimuli. Ann Otol Rhinol Laryngol;89:178-84.

10- Nagamine H, Iino Y, Kojima C, Miyazawa T. and Iida T. (2002): Clinical characteristics of so-called eosinophilic otitis media. Auris Nasus Larynx.; 29:19-28.

11- Okubo H, Ishikawa N. and Watanabe I. (1984):Sonotubometry [in Japanese; abstract in English]. Clinical Otolaryngologica (Kyoto).;77:1747-1754.

12- Ponikau JU, Sherris DA. and Dern EB. (1999): The diagnosis and incidence of allergic fungal sinusitis. Mayo Clin Proc.;74:877-884. 
13- Sadé J. and Ar A. (1997):Middle ear and auditory tube: Middle ear clearance, gas exchange and pressure regulation. Otolaryngol Head Neck Surg;116:499-524.

14- Settipane GA. (1996): Epidemiology of nasal polyps. Allergy Asthma Proc.;17:231-236.

15- Straetemans M, Van Heerbeek N, Tonnaer EL, Ingels K, Rijkers GT. and Zielhuis GA. (2001): A comprehensive model for the aetiology of otitis media with effusion. MedHypotheses.;57(6):784-791.

16- Suzuki H, Matsutani S. and Kawase T. (2004): Epidemiologic surveillance of "eosinophilic otitis media" in Japan [in Japanese; abstract in English]. Otol Japan:;14:112-117.

17- Tomioka S, Kobayashi T. and Takasaka T. (1997): Intractable otitis media in patients with bronchial asthma (eosinophilic otitis media). In: Sanna M, ed. Cholesteatoma and
Mastoid Surgery. Rome, Italy: Edizioni Internazionali CIC;: 851-853.

18- Tsuji T, Yamaguchi N. and Moriyama $H$. (2003): Patulous eustachian tube following otitis media [in Japanese; abstract in English]. Nippon Jibiinkoka Gakkai Kaiho.; 106:10231029 .

19- van Heerbeek N, Ingels KJ, Snik AF. and Zielhuis GA. (2001): Eustachian tube function in children after insertion of ventilation tubes. Ann Otol Rhinol Laryngol.;110 (12):1141-1146.

20- Virtanen H. (1978): Sonometry, an acoustical method for objective measurement of auditory tubal opening. Acta Otolaryngol (Stockh); 86:93-103.

21- Virtanen H. and Palva T. (1982): The patulous eustachian tube and chronic middle ear disease. Acta Otolaryngol;93:49 


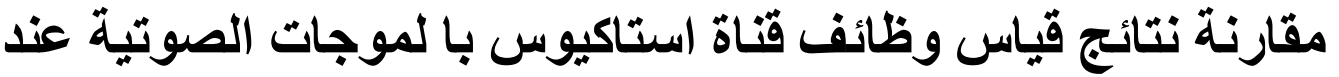

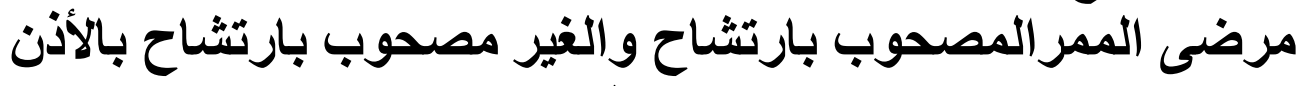 الوسطى
}

\author{
أحمد نصار - محمد عبد الله - محمد عطية
}

ان حدوث اي خلل في وظائف قناة استاكيوس قد يؤدى الى حدوث التهاب التهاب و ارتشاح بالأذن

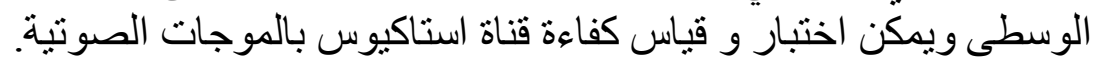

و هي طريقة من افضل الطرق ويمكن استخدامها في حالة وجود ثقب في طبلة الأذن او في الأذن السليمة.

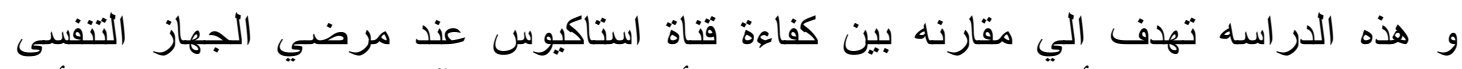

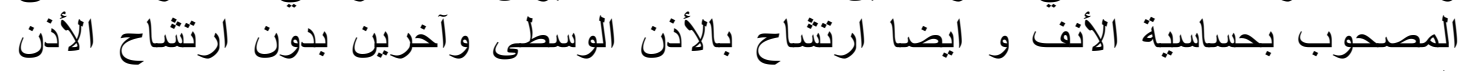

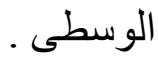

اجريت هذة الدر اسة علي 45 مريض قسمو ا الي ثلاثه مجمو عات و اثنتت النتائج ان زمن فتح قناة النقان

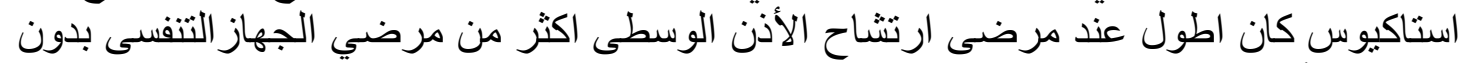

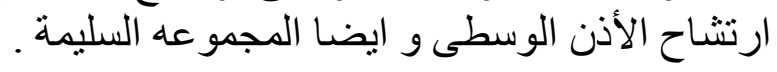

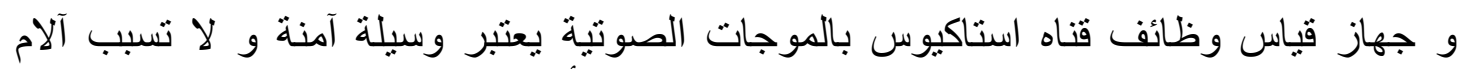

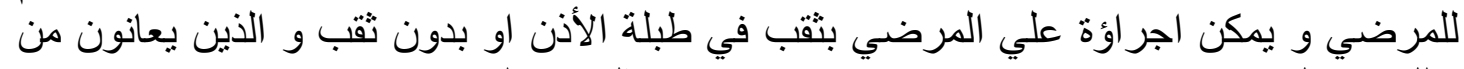

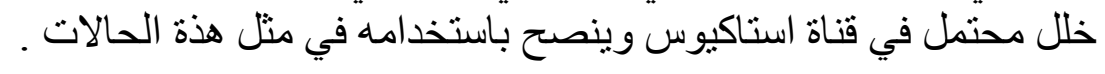

